\title{
Spatial filtering velocimetry of objective speckles for measuring out-of-plane motion
}

\author{
Jakobsen, Michael Linde; Yura, H. T.; Hanson, Steen Grüner
}

Published in:

Applied Optics

Link to article, DOI:

10.1364/AO.51.001396

Publication date:

2012

Document Version

Publisher's PDF, also known as Version of record

Link back to DTU Orbit

Citation $(A P A)$ :

Jakobsen, M. L., Yura, H. T., \& Hanson, S. G. (2012). Spatial filtering velocimetry of objective speckles for measuring out-of-plane motion. Applied Optics, 51(9), 1396-1406. https://doi.org/10.1364/AO.51.001396

\section{General rights}

Copyright and moral rights for the publications made accessible in the public portal are retained by the authors and/or other copyright owners and it is a condition of accessing publications that users recognise and abide by the legal requirements associated with these rights.

- Users may download and print one copy of any publication from the public portal for the purpose of private study or research.

- You may not further distribute the material or use it for any profit-making activity or commercial gain

- You may freely distribute the URL identifying the publication in the public portal

If you believe that this document breaches copyright please contact us providing details, and we will remove access to the work immediately and investigate your claim. 


\title{
Spatial filtering velocimetry of objective speckles for measuring out-of-plane motion
}

\author{
M. L. Jakobsen, ${ }^{1, *}$ H. T. Yura, ${ }^{2}$ and S. G. Hanson ${ }^{1}$ \\ 'DTU Fotonik, Department of Photonics Engineering, Technical University of Denmark, \\ P.O. Box 49, DK-4000, Roskilde, Denmark \\ ${ }^{2}$ Electronics and Photonics Laboratory, The Aerospace Corporation, P.O. Box 92957, \\ Los Angeles, California, 90009, USA \\ ${ }^{*}$ Corresponding author: mlja @ fotonik.dtu.dk
}

Received 29 August 2011; revised 10 November 2011; accepted 10 November 2011; posted 14 November 2011 (Doc. ID 153671); published 19 March 2012

\begin{abstract}
This paper analyzes the dynamics of objective laser speckles as the distance between the object and the observation plane continuously changes. With the purpose of applying optical spatial filtering velocimetry to the speckle dynamics, in order to measure out-of-plane motion in real time, a rotational symmetric spatial filter is designed. The spatial filter converts the speckle dynamics into a photocurrent with a quasi-sinusoidal response to the out-of-plane motion. The spatial filter is here emulated with a CCD camera, and is tested on speckles arising from a real application. The analysis discusses the selectivity of the spatial filter, the nonlinear response between speckle motion and observation distance, and the influence of the distance-dependent speckle size. Experiments with the emulated filters illustrate performance and potential applications of the technology. (C) 2012 Optical Society of America

OCIS codes: $\quad 030.1640,120.7250,030.1670,030.6140,030.6600,070.6110$.
\end{abstract}

\section{Introduction}

Speckle photography (SP) or digital speckle photography (DSP) [1,2] are well established techniques using the speckle dynamics to measure in-plane translation or deformation of an object. By adding a reference field, incident on the observation plane, speckle interferometry and SP or DSP can be combined to measure both the two in-plane and the out-of-plane components of movements [3] of an object surface. Typically, the speckle or interference patterns are acquired by a camera and then postprocessed with fringe analysis and/or correlation techniques to provide field measurements giving a high degree of spatial information.

The techniques of SP and DSP have also been combined with optical spatial filtering velocimetry (OSFV) [4] in order to carry out single-point mea-

1559-128X/12/091396-11\$15.00/0

(C) 2012 Optical Society of America surements of moving particles or rigid objects [5]. Such techniques can be integrated conveniently into a compact optical sensor [6], providing nearly realtime measurements of one or two components of an object in-plane motion [7]. The out-of-plane component can be addressed $\bar{b} y$ adding a reference field incident onto the observation plane, and designing multiple spatial filters that can deal with a compound intensity structure of random speckles and regular fringes, and thus give independent and simultaneous velocity measurements [8]. Such a velocity measurement technique is anisotropic in the sense that the out-of-plane motion will be resolved with a resolution that is one or two magnitudes higher than in the resolution for measurement of the two in-plane motions.

Speckle dynamics and OSFV have also been applied to measure the axial motion or the temporal distance from a sensor to the object. When objective speckles are observed at a distance $L$ from the object, the speckle dynamics, due to an in-plane object 
motion, will reflect the ratio $(L / R)$ of the distance of propagation $(L)$ to the radius $(R)$ of curvature of the phase front of the illuminating beam at incidence with the object plane $[\underline{9}, 10]$. Thus, by measuring the corresponding velocity of the speckles in two axially displaced observation plans $\left(L_{1}\right.$ and $\left.L_{2}\right)$, given the radius of curvature $R$, the absolute distance to the moving object can be determined in nearly real-time by using OSFV [11].

In this manuscript the covariance function of two intensity distributions acquired in the observation plane before and after an axial translation is determined in order to describe the radial speckle motions. Accordingly, a spatial filter is designed that can measure the radial motion of the speckles and distinguish whether the speckle pattern is expanding or contracting relative to the origin of the observation plane. With this filter we can directly measure the radial speckle motion as a response to the axial motion of an object. This is demonstrated experimentally, and finally possible applications are discussed. The purpose of this work is to demonstrate and study the possibility of using OSFV for measuring out-ofplane object motions in real-time.

\section{Theory}

In a Cartesian-coordinate coordinate system $s=$ $(x, y, z)$ we consider two parallel planes; the observation plane, which is fixed relative to the coordinate system and intersects the $z$-axis at $z$, and the object plane which moves parallel with the $z$-axis with a velocity of $v_{z}$. A position in the observation plane is described by the two-dimensional Cartesian coordinates, $\mathbf{p}=\left(p_{x}, p_{y}\right)$. A position in the object plane is described by the two-dimensional Cartesian coordinates, $\mathbf{r}=(x, y)$. Both coordinate systems share their respective origin with the $z$-axis at any time.

As illustrated in Fig. 1, a Gaussian beam propagates toward the object plane, parallel with the $z$-axis and with the field distribution centered on the $z$-axis.

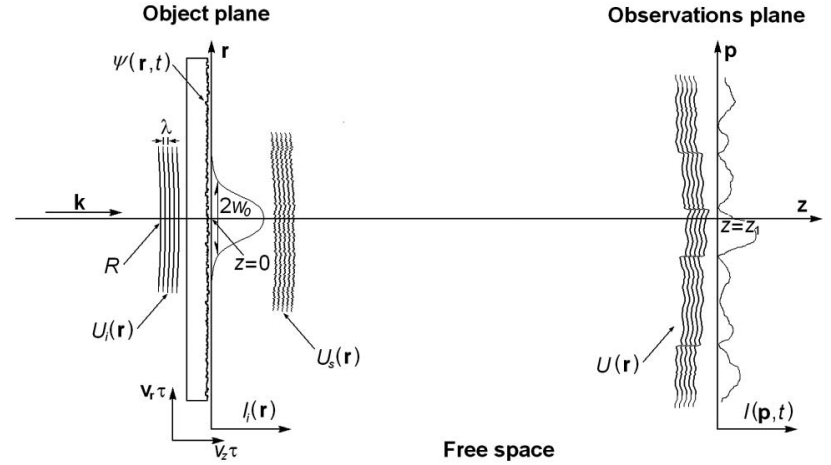

Fig. 1. Schematics of the setup used for the theoretical description. The object is considered a transparent diffuser.

An object moves along with the object plane, having a velocity given as: $\mathbf{v}(t)=\left(v_{x}, v_{y}, v_{z}\right)$. This means that the object can move in-plane relative to the object plane, described by a two-dimensional (2D) velocity vector, $\mathbf{v}_{r}=\left(v_{x}, v_{y}\right)$. The surface of the object is rough in the sense that its $\mathrm{rms}$ roughness is larger than $\lambda$, and that the diameter of the surface illumination is larger than any lateral scale of the surface roughness. Therefore, in the observation plane we observe an intensity distribution giving rise to fully developed speckles [12], described by the 2D Cartesian coordinates, $\mathbf{p}$. The intensity distribution is acquired at $t_{1}$, and again, after a time lag of $\tau$ at $t_{2}=t_{1}+\tau$. The shift in object position along the $z$ axis $\left(v_{z} \tau\right)$ during the time lag $\tau$ is considered so small that it does not change the parameters $\left(w_{0}, R\right)$ of the incident beam.

\section{A. Speckle Dynamics due to 3D Movement of an Object}

The dynamics of the speckles due to the movement of the object during the time lag can be described by the normalized spatio-temporal covariance function of the two acquired intensity distributions:

$$
C_{n}\left(\mathbf{p}_{1}, \mathbf{p}_{2} ; \tau\right)=\frac{\left\langle I\left(\mathbf{p}_{1}, t_{1}\right) I\left(\mathbf{p}_{2}, t_{1}+\tau\right)\right\rangle-\left\langle I\left(\mathbf{p}_{1}, t_{1}\right)\right\rangle\left\langle I\left(\mathbf{p}_{2}, t_{1}+\tau\right)\right\rangle}{\left\{\left[\left\langle I\left(\mathbf{p}_{1}, t_{1}\right)^{2}\right\rangle-\left\langle I\left(\mathbf{p}_{1}, t_{1}\right)\right\rangle^{2}\right]\left[\left\langle I\left(\mathbf{p}_{2}, t_{1}+\tau\right)^{2}\right\rangle-\left\langle I\left(\mathbf{p}_{2}, t_{1}+\tau\right)\right\rangle^{2}\right]\right\}^{1 / 2}},
$$

The case is constrained to $\left|v_{z}\right| \ll c$, the speed of light, where the field can be considered as quasi monochromatic with a wavelength of $\lambda$. The object plane intersects the Gaussian field, $U_{i}(\mathbf{r})$, at the position of $z=0$ at time $t_{1}$. In the object plane the Gaussian field is characterized by an $e^{-2}$ radius $w_{0}$ for the intensity distribution and a radius $R$ of curvature for the phase front of the field. where the angular brackets denote the ensemble average of the intensity distributions. In this paper we assume quasi-monochromatic conditions and stationary, isotropic statistical conditions for the object surface. In the object plane, and in the observation plane, the field is described using the complex optical scalar fields. In the observation plane, we expect that the complex scalar field has circular symmetric 
complex Gaussian statistics [12], in which case we can use

$$
C_{n}\left(\mathbf{p}_{1}, \mathbf{p}_{2} ; \tau\right)=\frac{\left|\Gamma\left(\mathbf{p}_{1}, \mathbf{p}_{2} ; \tau\right)\right|^{2}}{\Gamma\left(\mathbf{p}_{1}, \mathbf{p}_{1} ; 0\right) \Gamma\left(\mathbf{p}_{2}, \mathbf{p}_{2} ; 0\right)},
$$

where

$$
\Gamma\left(\mathbf{p}_{1}, \mathbf{p}_{2} ; \tau\right)=\left\langle U\left(\mathbf{p}_{1}, t_{1}\right) U^{*}\left(\mathbf{p}_{2}, t_{2}\right)\right\rangle
$$

is the mutual intensity of the optical field and the asterisk denotes the complex conjugate.

The optical scalar field $U(\mathbf{p}, t)$ in the observation plane can be expressed via the Green's function, $G(\mathbf{r}, \mathbf{p})$, as a function of the field, $U_{s}(\mathbf{r}, t)$, scattered at the target surface $(S)$ :

$$
U(\mathbf{p}, t)=\int_{S} \mathrm{~d}^{2} \mathbf{r} U_{s}(\mathbf{r}) G(\mathbf{r}, \mathbf{p} ; t),
$$

where the Green's function continuously varies according to the time-dependent distance between the object and the observation plane:

$$
\begin{aligned}
G(\mathbf{r}, \mathbf{p} ; t)= & -\frac{i k}{2 \pi\left(z-v_{z}\left(t-t_{1}\right)\right)} \\
& \times \exp \left(-\frac{i k}{2\left(z-v_{z}\left(t-t_{1}\right)\right)}\left(\mathbf{r}^{2}-2 \mathbf{r} \cdot \mathbf{p}+\mathbf{p}^{2}\right)\right)
\end{aligned}
$$

for $t \in\left[t_{1} ; t_{2}\right]$. Equation (5) describes the paraxial version of the Green's function [13] for free-space propagation. The length of the optical wave vectors is denoted as $k=2 \pi / \lambda$.

The transmitted optical field at the object plane is modeled as

$$
U_{s}(\mathbf{r})=U_{i}(\mathbf{r}, t) \Psi(\mathbf{r}, t),
$$

where $U_{i}(\mathbf{r})$ is the optical field, incident on the object plane, and $\Psi(\mathbf{r}, t)$ is the stochastic complex amplitude transmittance, which describes the surface of the diffuser. The statistics of the stochastic transmittance is assumed to be stationary and isotropic. Therefore, the speckles observed in the observation plane are fully developed speckles. However, the modulus $|\Psi(\mathbf{r}, t)|$ is assumed to be constant and set equal to unity. Following the analysis, described in [14], the correlation function for the amplitude transmittance can be found as

$$
\left\langle\Psi\left(\mathbf{r}_{1}, t\right) \Psi^{*}\left(\mathbf{r}_{2}, t\right)\right\rangle=\text { const. } \times \delta\left(\mathbf{r}_{1}-\mathbf{r}_{2}\right),
$$

i.e., the amplitude transmittance in a point is uncorrelated with the amplitude transmittance in any other point on the object, except for the point itself. Therefore, the mutual coherence function for the transmitted field follows as

$$
\begin{aligned}
\Gamma_{s}\left(\mathbf{r}_{1}, \mathbf{r}_{2} ; \tau\right)= & \left\langle U_{s}\left(\mathbf{r}_{1}, t_{1}\right) U_{s}^{*}\left(\mathbf{r}_{2}, t_{2}\right)\right\rangle \\
& \propto U_{i}\left(\mathbf{r}_{1}\right) U_{i}^{*}\left(\mathbf{r}_{2}\right) \delta\left(\mathbf{r}_{2}-\mathbf{r}_{1}{ }^{\prime}\right),
\end{aligned}
$$

where $\mathbf{r}_{1}^{\prime}$ is the position at time $t_{2}$ of the scatterer on the object surface that was located at position $\mathbf{r}_{1}$ at time $t_{1}$. Further, an unimportant constant value has been discarded.

Inserting Eqs. (4) and (8) into Eq. (3), the mutual coherence function of the optical field obtained at the observation plane becomes

$\Gamma\left(\mathbf{p}_{1}, \mathbf{p}_{2} ; \tau\right)=\int_{S} \mathrm{~d}^{2} \mathbf{r} U_{i}(\mathbf{r}) U_{i}^{*}\left(\mathbf{r}^{\prime}\right) G\left(\mathbf{r}, \mathbf{p}_{1} ; t_{1}\right) G^{*}\left(\mathbf{r}^{\prime}, \mathbf{p}_{2} ; t_{1}+\tau\right)$,

where similarly to $\mathbf{r}^{\prime}=\mathbf{r}+v_{x y} \tau$, the Greens function becomes $G^{\prime}\left(\mathbf{r}^{\prime}, \mathbf{p} ; t\right)=G\left(\mathbf{r}+v_{x y} \tau, \mathbf{p} ; t+\tau\right)$.

The stationary, complex optical scalar field $U_{i}(\mathbf{r})$ incident onto the object is, to within an unimportant multiplicative factor:

$$
U_{i}(\mathbf{r})=\exp \left(-\left(x^{2}+y^{2}\right)\left(\frac{1}{w_{0}^{2}}+\frac{i k}{2 R}\right)\right) .
$$

The sign convention used for the curvature is such that a converging illuminating beam corresponds to $R<0$, whereas a diverging beam corresponds to $R>0$. Substituting Eqs. (ㅁ) and (10) into Eq. (9), performing the resulting Gaussian integrations, and simplifying yields:

$$
\begin{aligned}
C_{n}\left(\mathbf{p}, \Delta \mathbf{p}, v_{z} ; \tau\right)= & \frac{\exp \left(-\left(\frac{\mathbf{v}_{x y} \tau}{w_{0}}\right)^{2}\right)}{\left(\left(1+\frac{v_{z} \tau}{z}\right)^{2}+\left(\frac{v_{z} \tau}{l_{z}}\right)^{2}\right)} \\
& \times \exp \left(-\frac{1}{\rho_{z}^{2}}\left[\Delta \mathbf{p}-\left(1+\frac{z}{R}\right) \mathbf{v}_{x y} \tau\right.\right. \\
& \left.\left.+\frac{v_{z} \tau}{z}\left(\mathbf{p}+\left(\frac{1}{2}+\frac{z}{R}\right) \mathbf{v}_{x y} \tau\right)\right]^{2}\right),
\end{aligned}
$$

where

$$
\rho_{z}^{2}=\rho_{0}^{2}\left(\left(1+\frac{v_{z} \tau}{z}\right)^{2}+\left(\frac{v_{z} \tau}{l_{z}}\right)^{2}\right)
$$

and

$$
\rho_{0}^{2}=\frac{8 z^{2}}{k^{2} w_{0}^{2}} \quad \text { (mean speckle radius) }
$$

$$
l_{z}=\frac{4 z^{2}}{k w_{0}^{2}} \quad \text { (mean speckle length) }
$$

As presented in Eq. (11), the covariance function consists of two major factors: The first factor describes the decorrelation of the correlation peak as 
a function of in-plane and out-of-plane movement of the object. The second factor describes the position of the correlation peak as a function of the threedimensional (3D) movement of the object during the time lag $\tau$.

In case the object carries out an in-plane movement of $\mathbf{v}_{\mathbf{x y}} \tau$ during the time lag $\tau$ (i.e., $v_{z}=0$ ), the position of the correlation peak describes a similar in-plane translation of the speckles in the observation plane. The relation between movement of the speckles and object is specified as $\Delta \mathbf{p}=(1+z / R) \mathbf{v}_{\mathbf{x y}} \tau$, as it can be found in $[9,10,14]$. The decorrelation length of the speckles equals the radius $w_{0}$ of the beam at incidence at the object.

In case the object carries out a movement along the $z$-axis only (i.e., $\left|\mathbf{v}_{\mathbf{x y}}=0\right|$ ), we acquire the first series of intensities at $t_{i}$ for the ensemble-average covariance function at position $\mathbf{p}=\left(p_{x}, p_{y}\right)$ given the initial object distance $z$. Then, moving the object a distance of $v_{z} \tau$ and acquiring the second series of intensities at $t_{i}+\tau$, the position of the correlation peak can be found from Eq. (11) for $\left|\mathbf{v}_{\mathbf{x y}}=0\right|$ as:

$$
\Delta \mathbf{p}=-\frac{v_{z} \tau}{z} \mathbf{p} .
$$

Therefore, in the observation plane the speckles will move along a straight line defined by the origin and the initial position of observation (p). In the Cartesian-coordinate system, the position of the correlation peak can be found at the point of intersection between the observation plane and a straight line locked to the origin of the object plane-i.e., the center of the illuminated area. As mentioned earlier, the origin of the object plane defines the intersection between the object and the center of the incident field $\left(U_{i}(\cdot)\right)$. Further, the orientation of this straight line is fixed, and it can be defined from the initial point (p) of observation at $\mathbf{S}_{\mathbf{P}}=\left(p_{x}, p_{y}, z\right)$ and the initial position $\mathbf{S}_{\mathbf{C}}=(0,0,0)$ of the origin of the object plane.

Typically, $l_{z}^{2} \gg \rho 0^{2}$, therefore, on average, 3D speckles tend to be elongated structures which are orientated along straight lines, which all originate from the origin of the object plane and point in any direction toward the observation plane. As the object and the object plane move along the $z$-axis, the 3D speckles will move along, as long as the beam parameters in the object plane remain constant. This is in full agreement with predictions in the literature $[15,16]$ for a static $\left(\left|\mathbf{v}_{\mathbf{x y}}=0\right|\right)$ object, where the observation plane is scanned along the $z$-axis instead. However, as we shall come back to later, the individual 3D speckles are not aligned exactly with these lines.

Therefore, in case $v_{z}<0$, the distance between the object and the observation plane increases with time $t$, and accordingly the correlation function will measure a radial movement of the speckles away from the origin of the observation plane. A reversal of the $z$-velocity will reverse the radial displacement of the speckles. According to Eq. (15), the radial speckle speed in response to a certain out-of-plane movement of the object will increase proportional to the modulus of p. Further, considering out-of-plane movements, which are comparable to the initial $z$ values, the nonlinear dependency of $z$ on the response will become apparent.

In case the object carries out a movement which combines both an in-plane movement of $\mathbf{v}_{\mathbf{x y}} \tau$ and an out-of-plane movement of $v_{z} \tau$ during the time lag $\tau$, and given an arbitrary position $\mathbf{p}$ of observation, the peak of the correlation function describes a shift in the speckle pattern at observation point p by $\Delta \mathbf{p}$ :

$$
\Delta \mathbf{p}=\mathbf{v}_{x y} \tau-\frac{v_{z} \tau}{z}\left(\mathbf{p}+\frac{1}{2} \mathbf{v}_{x y} \tau\right),
$$

where we, for simplicity, have assumed that $R \rightarrow \infty$. The line describing the position of the peak correlation as a function of $v_{z} \tau$ does not point toward the origin of the object plane, as it was the case for $\left|\mathbf{v}_{\mathbf{x y}}=0\right|$. Neither does the line follow the usual inplane speckle motion found in Eq. (11) as $\Delta \mathbf{p}=(1+z / R) \mathbf{v}_{\mathbf{x y}} \tau$. Instead, the line points toward the position at $\mathbf{S}_{\mathbf{C}}=\left(v_{x} \tau / 2, v_{y} \tau / 2,0\right)$ in the object plane, and a line through any other observation point does the same. This is in full agreement with the prediction for dynamic speckles given by [15]. However, this result is not in agreement with [1] $]$, where the $1 / 2$ factor in Eqs. (11) and (16) is replaced with the incorrect factor of 1 . Note that even though the lines of position of peak correlation functions do not follow the usual in-plane speckle motion found in Eq. (11) as $\Delta \mathbf{p}=(1+z / R) \mathbf{v}_{\mathbf{x y}} \tau$, so do the individual 3D speckles indeed. The difference appears because the covariance function is based on ensemble averaging many $3 \mathrm{D}$ speckles, and they point at random positions within the illuminated spots on the object surface.

\section{B. Spatial Filter}

Generally, in optical spatial filtering velocimetry [4], a dynamic intensity distribution $I$ (p) illuminates a spatial filter with an intensity transmittance of $t(\mathbf{p}-\mathbf{q})$ and produces a resulting photocurrent $i(\mathbf{q})$ of a value, depending on the position of the intensity distribution relative to the filter. The filter will be located in the observation plane $\mathbf{p}$, and the displacement of the intensity distribution across the filter is described by $\mathbf{q}$ :

$$
i(\mathbf{q})=\int_{-\infty}^{\infty} \mathrm{d} \mathbf{p} I(\mathbf{p}) t(\mathbf{p}-\mathbf{q}) .
$$

In case optical spatial filtering velocimetry is applied to a one-dimensional, in-plane translation of an intensity distribution relative to the filter [5], the intensity transmittance $t(\mathbf{p})$ for the optimum spatial filter will have a one-dimensional repetitive structure. Therefore, the intensity transmittance obeys that $t(\mathbf{p})=t(\mathbf{p}+\Lambda)$ where the direction of $\Lambda$ defines the direction of the displacement or velocity 
component to be measured, and $|\Lambda|$ defines the structural period of the intensity transmittance. For instance, the intensity transmittance could be a cosine function. Then, the first derivative of the spatial phase of the intensity transmittance along the direction of $\Lambda$ will be constant. Typically, an additional filter having the same transmission function, though shifted $\pi / 2$ relative to the first filter, is added in spatial filtering velocimetry to produce two photocurrents in phase quadrature. Both filters observe the same speckle pattern, thus the two photocurrents are strongly correlated, and the direction of the speckle motion can be found.

However, as described in Eq. (15) the speckle motion we intend to measure in this paper is moving in radial direction away or toward the center of the observation plane, and with a velocity given at a point in the observation plane which is proportional to the radial distance $|\mathbf{p}|$. Therefore, a structured detector arrangement, which is rotationally symmetric with regard to the center of the observation plane, is required. In principle, a pair of detectors organized as coinciding rings will provide two signals with a mutual time delay, which can be extracted with crosscorrelation function. However, in order to gain a higher selectivity of the spatial filter [4], additional pairs of detector rings must be added to the structure. Further, in order to match the positiondependent gain of the speckles movement, Eq. (15), the filter period, as a function of radius $r_{\mathrm{p}}$ in the $\mathrm{ob}-$ servation plane, must increase linearly with the $r_{\mathrm{p}}$. Therefore, the first derivative of the phase $\theta\left(r_{\mathrm{p}}\right)$ with respect to $r_{\mathrm{p}}$ can be written as:

$$
\frac{d \theta\left(r_{\mathrm{p}}\right)}{d r_{\mathrm{p}}}=\frac{2 \pi \alpha}{r_{\mathrm{p}}}
$$

where $\alpha$ is a constant. Equation (18) is reorganized and integrated in order to derive $\left.\overline{\theta(}_{\mathrm{p}}\right)$ :

$$
\int_{\theta_{0}}^{\theta} \mathrm{d} \theta\left(r_{\mathrm{p}}\right)=2 \pi \alpha \int_{r_{0}}^{r} \frac{\mathrm{d} r_{\mathrm{p}}}{r_{\mathrm{p}}}
$$

where the boundary condition, $\theta_{0}=\theta\left(r_{0}\right)$, defines the (arbitrary) absolute phase. We find:

$$
\theta\left(r_{\mathrm{p}}\right)-\theta_{0}=2 \pi \alpha \ln \left(\frac{r_{\mathrm{p}}}{r_{0}}\right) .
$$

And finally, the complete intensity transmission function for the filter becomes:

$$
t\left(r_{\mathrm{p}}\right)=\cos \left(2 \pi \alpha \ln \left(\frac{r_{\mathrm{p}}}{r_{0}}\right)+\theta_{0}\right) .
$$

Applying a second filter similar to Eq. (21), though inserting $\theta_{0} \rightarrow \theta_{0}+\pi / 2$ instead, two photocurrents in mutual phase quadrature will be available and thus provide information on to what extent the speckle pattern is expanding or contracting.
Figure 2 shows the intensity transmission function of the filter specified in Eq. (21) within the range of $r_{\mathrm{p}} \in[1,512]$, and for $r_{\mathrm{p}}>512$ the transmission function equals 0 . The brightest level indicates a filter value of one, while the darkest level indicates a filter value of minus one. Approaching the origin of the observation plane, the spatial frequency of the periods of the filter function will increase beyond any practical use for the application (see Section 4 for more details) or ultimately it will be limited by the resolution of the medium implementing the filter.

The rotational symmetric power spectrum of the filter function can be found as a function of $1 / r_{\mathrm{p}}$ by using the Fourier-Bessel transform. In Fig. 3, the power of the Fourier-Bessel transform of the filter function illustrated in Fig. 2 is plotted (see Appendix A for an analytic solution). The discrete and nonequidistant spatial frequencies present in the filter function are apparent. Considering fullydeveloped speckles, the spatial density, size and intensity of the speckles throughout the observation plane are uniform. Therefore, for a given object position, Fig. 3 illustrates that the contributions to the signal in the range of spatial frequencies from 0.020 to 0.028 are weighted higher than the contributions from the rings responsible for spatial frequencies higher than 0.028 . Generally, this phenomenon will cause the phase-shifted filter outputs to be mutually unbalanced in terms of their lowfrequency contents.

Generically, the two photocurrents can be balanced without affecting the filter signals by defining an even number of angular sectors in the transmission function, where the phases between adjacent sectors

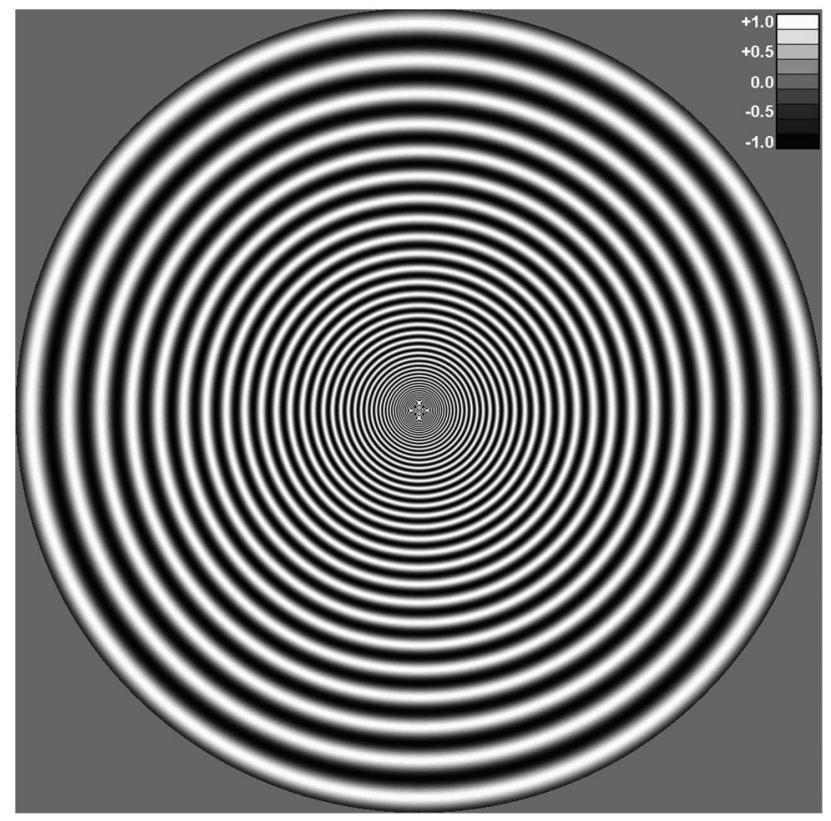

Fig. 2. Intensity transmission function in Eq. (21) plotted as a function of radius in the observation plane. The plot uses the following parameters; $r_{0}=1, \theta_{0}=0, \alpha=10$ and for the range of $r_{\mathrm{p}} \in[1 ; 512]$. For $r_{\mathrm{p}}>512$ we set $t\left(r_{\mathrm{p}}\right)=0$. 
are shifted $\pi$ mutually. Letting $\varphi$ denote the angular coordinate of the polar coordinate system, the new transmission function becomes:
This consideration is the same for a linear filter, and the higher the selectivity of the filter is, the more oscillations the average burst will contain, and the

$$
t\left(r_{\mathrm{p}}\right)=\left\{\begin{array}{ll}
\cos \left(2 \pi \alpha \ln \left(\frac{r_{\mathrm{p}}}{r_{0}}\right)+\theta_{0}\right), & \text { for } \frac{\pi}{2} n<\varphi \leq \frac{\pi}{2}\left(n+\frac{1}{2}\right), n=-2,-1,0,1 \\
\cos \left(2 \pi \alpha \ln \left(\frac{r_{\mathrm{p}}}{r_{0}}\right)+\pi+\theta_{0}\right), & \text { for } \frac{\pi}{2}\left(n+\frac{1}{2}\right)<\varphi \leq \frac{\pi}{2}(n+1), n=-2,-1,0,1
\end{array} .\right.
$$

In Fig. 4 the transmission function in Eq. (22) is illustrated. In this case the number of sectors is eight.

Given the distance $(z)$ between the object and the observation plane, the speckle statistics provide a constant speckle size and speckle density throughout the observation plane. However, in case the distance increases, the speckle pattern expands, and while the speckles slowly decorrelate, the size of the individual speckles increase to fill out the expanding speckle pattern, accordingly. The mutual distance between the speckles will change as anticipated by the filter design. Thus, as the object distance increases, the average number of speckles leaving an arbitrary ring $i$ at radius $r_{i}$ will be the same as the average number of speckles entering the next ring $i+1$ at radius $r_{i}+$ 1 for $r_{i}+1>r_{i}$. Therefore, the groups of speckles initially interrogated by the different rings will move consistently from one ring to the next as the object distance increases or decreases. In other words, the selectivity of this filter depends on either the number of rings, contributing to the signal, or speckle decorrelation, in this specific case defined by the mean speckle length $l_{z}$.

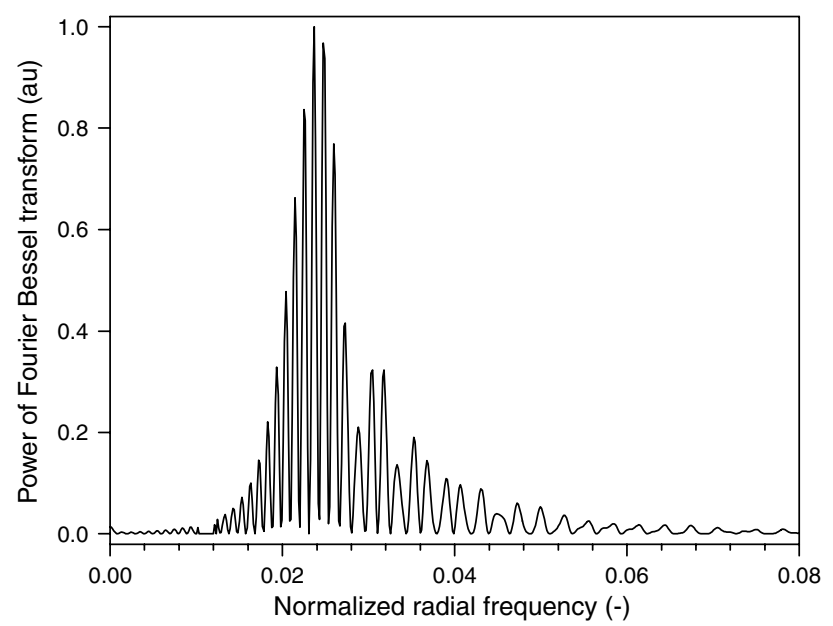

Fig. 3. Power of the Fourier-Bessel transform of the transmission function in Fig. 2 plotted as a function of radial frequency, normalized with the sample frequency. smaller the random measurement errors will be. However, by increasing the selectivity with regard to radial speckle movement, the sensor at the same time becomes less tolerant with respect to simultaneous in-plane motion.

\section{Experiments and Results}

As illustrated in Fig. 5, a HeNe laser provides the coherent illumination of the object, through an aperture. The aperture only facilitates alignment of the setup. The laser emits light at a wavelength of $\lambda=$ $632 \mathrm{~nm}$ and the beam is effectively collimated through the setup and provides an illuminating spot with a radius of $w_{0}=0.81 \mathrm{~mm}$ at the object, which is a transparent glass plate. The surface facing away from the laser is glass-blown to an extent that it produces fully developed speckles in the observation

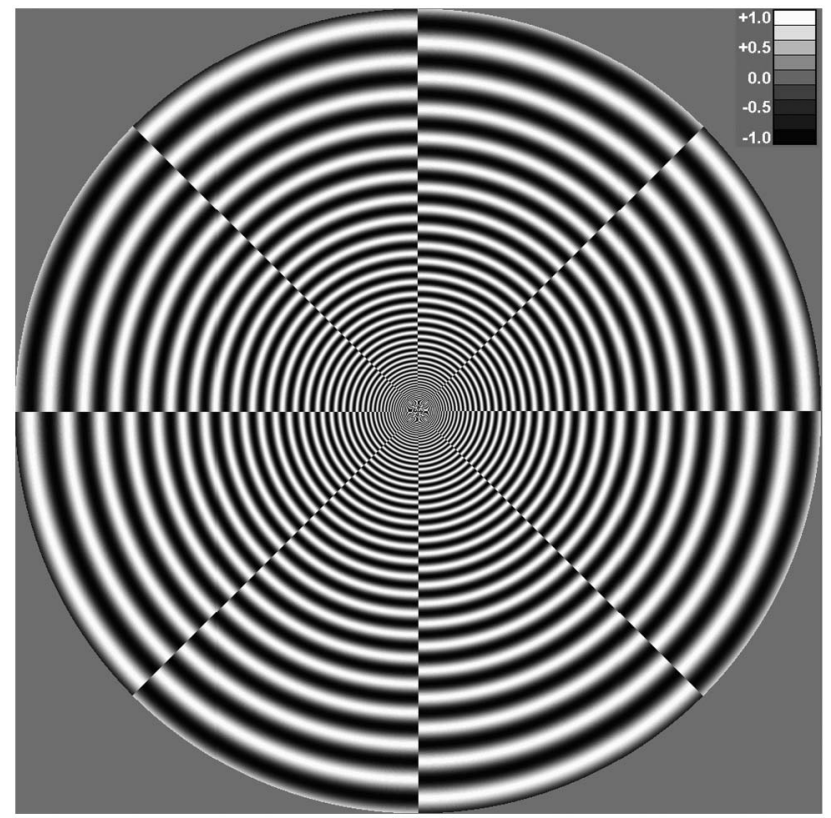

Fig. 4. Intensity transmission function in Eq. (22) plotted as a function of radius in the observation plane. The plot uses the following parameters; $r_{0}=1, \theta_{0}=0, \alpha=10$ and for the range of $r_{\mathrm{p}} \in[1 ; 512]$. For $r_{\mathrm{p}}>512$ we set $t\left(r_{\mathrm{p}}\right)=0$. 


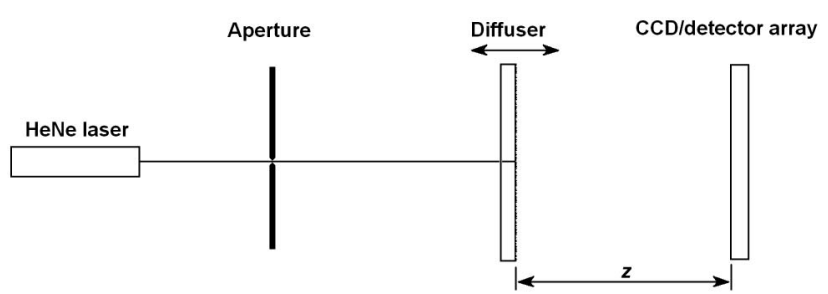

Fig. 5. Schematics of the experimental setup are illustrated. The object is a glass plate with a diffuse surface facing the CCD camera.

plane. The object is mounted on a motorized micrometer stage, which displaces the object along the optical axis in steps of $\Delta_{z}=-0.01 \mathrm{~mm}$ with a total displacement of $23.0 \mathrm{~mm}$. Note that in this experiment the object moves toward the aperture. From the object surface, scattered light propagates through a distance of $z$ to the observation plane, where a CCD camera is located. The CCD camera acquires black and white images of the speckles with a resolution of $1024 \times 1280$ pixels. The distance between the pixels in the camera is $5.2 \mu \mathrm{m} \times 5.2 \mu \mathrm{m}$. The electronic noise is reduced by ensemble averaging five images for each acquisition. In the experiment, any lateral motion of the illuminating spot as it translates axially is sought to be minimized. Therefore, the alignment of the CCD relative to the incidence of the beam coming directly from the laser beam is critical. The object is removed temporally, and normal incidence of the laser beam onto the CCD is achieved by directing its reflection from the CCD array back through the setup $(300 \mathrm{~mm})$ and back through a $0.5 \mathrm{~mm} \varnothing$ hole in the aperture. The experiment starts with a distance between object and the CCD of $z=(25.4+23.0) \mathrm{mm}$, while the experiment ends at a distance of $25.4 \mathrm{~mm}$. Therefore through the experiment, the speckle pattern collapses, and the mean speckle radius decreases from $\rho_{0}=17 \mu \mathrm{m}$ to $\rho_{0}=8.9 \mu \mathrm{m}$ as the object moves from position $z=48.4 \mathrm{~mm}$ to $z=25.4 \mathrm{~mm}$, respectively. The corresponding lengths $l_{z}$ of the speckles as 3D structures [Eq. (14)] become $1.4 \mathrm{~mm}$ and $0.4 \mathrm{~mm}$, respectively. Thus, the individual steps $(\Delta z)$ along the optical axis are significantly smaller than the mean speckle lengths, while the total travel is significantly larger. For each step, a speckle pattern is acquired with the CCD camera and saved for later postprocessing.

To illustrate the speckle motion as a function of the out-of-plane motion of the object, the speckle images are processed using speckle-correlation techniques. For the object position $(z)$ the corresponding speckle image is divided into $7 \times 9$ interrogation areas of $256 \times 256$ pixels and separated by $128 \times 128$ pixels. Each interrogation area is cross-correlated with the interrogation area located at the same position in the speckle image acquired at the object position $(z-50 \Delta z)$, respectively. A combined peak search in the correlation function, and a determination of the centroid position within the top $90 \%$ of the correla- tion peak, provide a vector in the observation plane. This vector is a local measurement in the observation plane of the speckle shift, occurring due to the movement of the object from position $z$ to position $z-50 \Delta z$. Plotting all the vectors obtained from two images acquired in the observation plane at object positions of $z=54.5 \mathrm{~mm}$ and $z-50 \Delta z=$ $55.0 \mathrm{~mm}$ (data from different experiment where $\left.v_{z} \tau<0\right)$ the vector map, illustrated in Fig. 6 appears. The speckle pattern is expanding, thus all the vectors point away from a position which approximately coincides with the center of the image, and the lengths of the individual vectors are proportional to their distance to the center of the image, as it is predicted in Eq. (15).

The center of expansion is estimated by determining the first order of momentum of the reverse vector lengths:

$$
r_{c}\left(r^{\prime}, c^{\prime}\right)=\frac{\left(\sum_{r=1}^{1024} \sum_{c=1}^{1280} \frac{r}{|\Delta \mathbf{p}(r, c)|^{2}}, \sum_{r=1}^{1024} \sum_{c=1}^{1280} \frac{c}{|\Delta \mathbf{p}(r, c)|^{2}}\right)}{\sum_{r=1}^{1024} \sum_{c=1}^{1280} \frac{1}{|\Delta \mathbf{p}(r, c)|^{2}}} .
$$

Processing the images acquired within the relevant range of positions, and determining the centers of all the corresponding maps, we find that throughout the full range of $23.0 \mathrm{~mm}$ the average center of the expansion is located at $r_{c, \text { all }}=(549.5,598.3)$ with a systematic error of less than 1 pixel, and a standard deviation of 4.5 and 8.2 pixels in vertical and horizontal direction, respectively. The estimate of the average center $\left(r_{c, \text { all }}\right)$ of expansion or contraction for a given experiment then defines the center for the computer generated spatial filter and will be applied to the data acquired in the same experiment.

The spatial filtering velocimetry is implemented by postprocessing the speckle images in a computer.

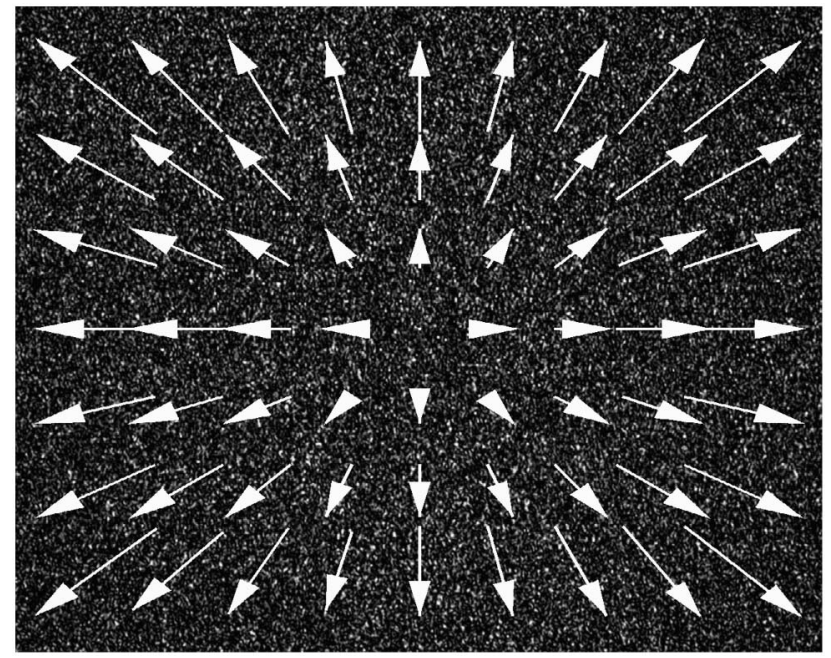

Fig. 6. Vector map is obtained by measuring the local speckle movement as the object moves away from the observation plane from position $z=54.5 \mathrm{~mm}$ to $z+\Delta z=55.0 \mathrm{~mm}$. 
The speckle image acquired at object position $z$ is denoted as $I_{s p}(r, c ; z)$, where $(r, c)$ address the individual pixels in the image. The filter functions are implemented as electronic images $s_{\theta}(r, c)$, having the same resolution as the speckle images of $1024 \times 1280$ cells. The index indicates the initial phase $\theta\left(r_{0}\right)$ of the filter at $r_{\mathrm{p}}=r_{0}$. During processing, the speckle image acquired at the object position $z$, each pixel in the speckle image is multiplied with the corresponding pixel in the filter image, and all the products are added together to produce a photocurrent $i_{\theta}(z)$ :

$$
i_{\theta}(z)=\sum_{r=1}^{1024} \sum_{c=1}^{1280} I_{s p}(r, c ; z) s_{\theta}(r, c) .
$$

Stepping through all speckle images, a complete record of the photocurrent $i_{\theta}(z)$ can be plotted as a function of object position $z$.

In Fig. 7(a) the photocurrents of $i_{0}(z)$ and $i_{\pi / 2}(z)$ are plotted as a function of $z$, for the filter function described in Eq. (21) for $\alpha=40, \theta_{0}=0$. Clearly the individual photocurrents oscillate with a quasi-sinusoidal dependency of $z$, and the two plots are shifted by a phase difference of $\pi / 2$. The sign of the phase shift between the two photocurrents changes as the direction of object movement changes. Apparently, the envelopes of the two photocurrents are different and drift apart throughout the relevant range of $z$ values. Figure 7(b) shows the photocurrents of $i_{0}(z)$ and $i_{\pi / 2}(z)$ for the filter function described in Eq. $(22)\left(\theta_{0}=0\right)$, and applied to the same speckle images as in Fig. 7(a). In this case, clearly the two photocurrents are balanced throughout the relevant range of $z$-values. For that reason, the filter function in Eq. (22) will be used throughout the rest of the experiments.

In order to determine long-range displacements throughout many oscillations in the photocurrents, the phase of the oscillations is determined as the angle of the phasor from the polar form of the two signals in mutual phase quadrature. However, the $z$-dependency in Eq. (15) makes such applications more circumstantial. More details on this can be found in the discussion.

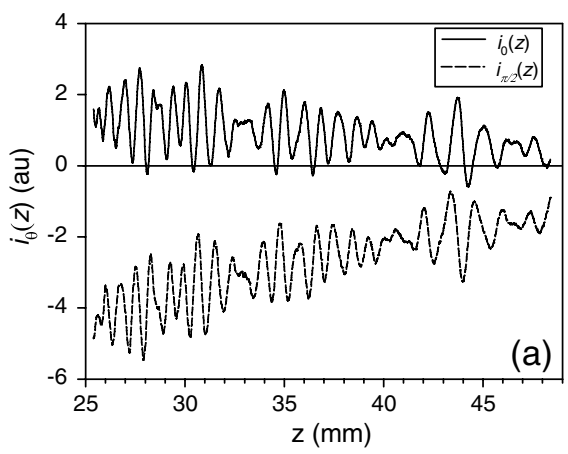

To accommodate the application for small-range displacement or for vibration sensing, we select a range of $z$, within which the responses for both signals $i_{0}(z)$ and $i_{\pi / 2}(z)$ provide well defined oscillations. According to [17] the probability of random phase changes is conditioned strongly by the amplitude of the signal. In case of high-signal amplitudes, the probability for a random phase change becomes very low, thus for this application a suitable $z$-position with high-signal amplitude is chosen at $z=28.9 \mathrm{~mm}$. Oscillating motions with three different amplitudes are generated with the motorized stage: $0.015 \mathrm{~mm}$, $0.15 \mathrm{~mm}$, and $2.0 \mathrm{~mm}$. The smallest amplitude is significantly smaller than the actual mean speckle length $\left(l_{z} \sim 0.15 \mathrm{~mm}\right)$ at $z=28.9 \mathrm{~mm}$, while the largest amplitude is significantly larger. In order to make the measurements independent of the oscillatory response function of the filter, again the displacement is determined as the angle of the phasor from the polar form of the two photocurrents in mutual phase quadrature.

In Fig. 8(a) the measurements of an oscillating motion is plotted as a function of steps of the stage. The amplitude for the motions is set to $0.15 \mathrm{~mm}$. The corresponding power spectrum is plotted in Fig. 8(b) and readings of the peak power in the harmonics provide a distortion factor of 0.016 . The background is established $40 \mathrm{~dB}$ below the signal level, thus, the noiseequivalent power corresponds to an amplitude of $1.5 \mu \mathrm{m}$. In Table 1 the data obtained for all three cases are listed.

\section{Discussion}

Equation (15) shows that the response to out-ofplane object motion on the speckle motion depends on both the position (p) of observation in the observation plane, and the distance $(z)$ between the object and the observation plane. The spatial filters described in Eq. (22) corrects for the dependency regarding p. However, moving the object through an axial displacement that is compatible with the distance $z$, the dependency of $1 / z$ will appear as a nonlinear effect in the measurements of $v_{z} \tau$. In Fig. 9 the displacement is measured as the angle of the phasor from the polar form of the two photocurrents and

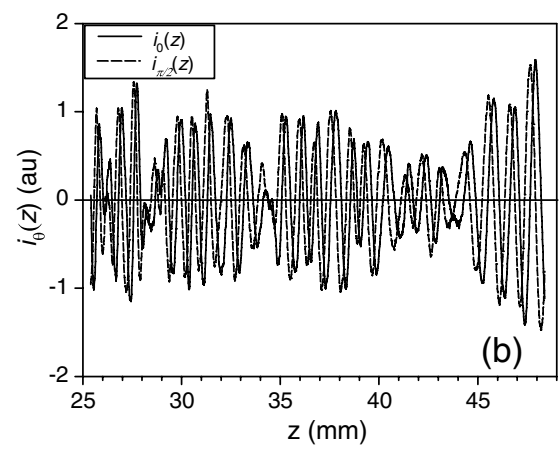

Fig. 7. Photocurrents of $i_{0}(z)$ and $i_{\pi / 2}(z)$ are plotted as a function of object positions $z$. Left plot (a) illustrates the filter function described in Eq. (21), while right plot (b) illustrates the filter function described in Eq. (22). The common parameters for the filters are; $\alpha=40$ for $r_{\mathrm{p}} \in[1 ; \overline{51} 2]$, while $t\left(r_{\mathrm{p}}\right)=0$ for $r_{\mathrm{p}}>474$. 

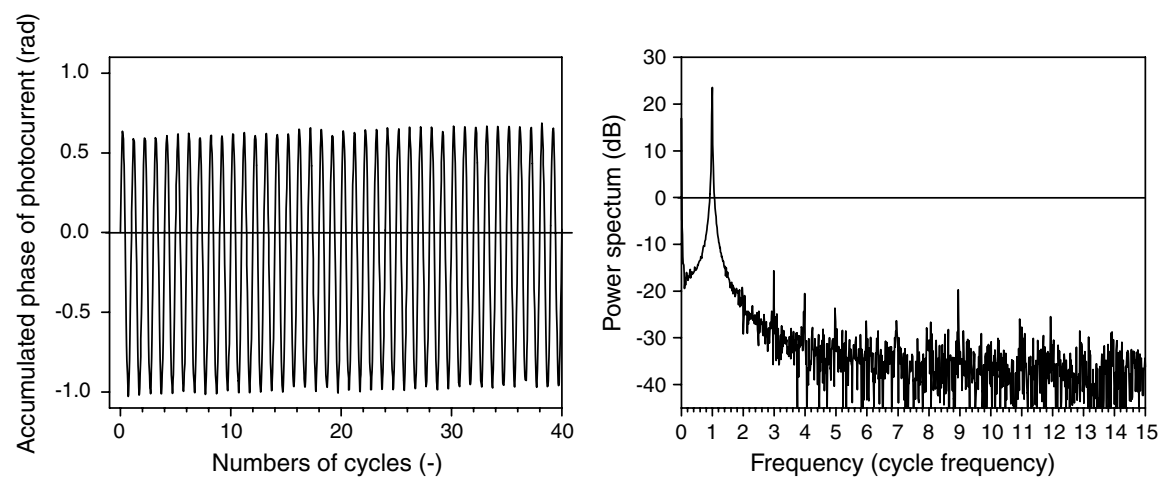

Fig. 8. To the left, the measured displacement of the object is plotted as a function of steps of the stage. The amplitude of the oscillation is $0.15 \mathrm{~mm}$. To the right, the power spectrum obtained for 67 oscillations is plotted. The filter [Eq. (22)] parameters are; $\alpha=40$ for $r_{\mathrm{p}} \in[1 ; 512]$, while $t\left(r_{\mathrm{p}}\right)=0$ for $r_{\mathrm{p}}>474$.

plotted as a function of the distances $z$. Clearly, the curve deviates from a straight line as a function of $z$.

As the object moves from the starting position $\left(z_{1}=48.4 \mathrm{~mm}\right)$ to position $z$, the filter tracks a selected spatial speckle structure contracting by $|\Delta \mathbf{p}|$ in the radial direction with regard to the origin of the observation plane. The selected speckle structure is defined by Eq. (18), thus, the phasor angle changes as function of radial speckle movement $|\Delta \mathbf{p}|$ just as fast as the filter changes phasor as a function of $d r_{\mathrm{p}}$. In the observation plane, $r_{\mathrm{p}}$ can substitute $|\mathbf{p}|$ directly, and Eq. (15) provides that $|\Delta \mathbf{p}| /|\mathbf{p}|=d z / z$ for small steps of $\Delta \bar{z}$. Finally, the corresponding change in phasor angle for the photocurrents becomes:

$$
d \theta=\frac{2 \pi \alpha}{r_{\mathrm{p}}} d r_{\mathrm{p}}=\frac{2 \pi \alpha}{z} d z
$$

After integrating from $z_{1}$ to $z$, the phasor angle for the photocurrents as a function of $z$ becomes

$$
\theta(z)=2 \pi \alpha \ln \left(\frac{z_{1}}{z}\right)
$$

where the boundary of $\theta(z)$ is set to zero at $z=z_{1}$. Plotting Eq. (26) in Fig. 9, the overall trend of the measured curve agrees with the theoretical curve within the random deviations. The standard deviation of the difference between the measured curve and theoretical curve predicted by Eq. (26) is approximately of $\pm 2.8 \mathrm{rad}$, and corresponds to a displacement of approximately $0.5 \mathrm{~mm}$. Therefore, the technology could be addressed to application where the range of displacements is either much larger than, or less than, the mean speckle length. In case of long range measurements, two devices located with a known difference in distance to the object

Table 1. Data and Measurements on Oscillating Motions

\begin{tabular}{cccc}
\hline $\begin{array}{c}\text { Amplitude of } \\
\text { oscillation }(\mathrm{mm})\end{array}$ & $\begin{array}{c}\text { Step size } \Delta z \\
(\mathrm{~mm})\end{array}$ & $\begin{array}{c}\text { Distortion } \\
\text { factor }(-)\end{array}$ & $\begin{array}{c}\text { Rel. background } \\
\text { noise }(\mathrm{dB})\end{array}$ \\
\hline 0.015 & 0.001 & 0.16 & -35 \\
0.15 & 0.01 & 0.016 & -40 \\
1.0 & 0.03 & 0.018 & -60 \\
\hline
\end{tabular}

could measure the axial motion of the object simultaneously. Then, the nonlinear effect could provide an estimate of the absolute distance to the object and compensate the measurements for the influence of the nonlinear effect accordingly.

In Fig. 10 the power of the Fourier-Bessel transform of the speckle patterns is plotted for two distances of the object, $z_{1}$ and $z_{2}$, where $z_{1}=25.4 \mathrm{~mm}$ and $z_{2}=48.4 \mathrm{~mm}$. The power of the Fourier-Bessel transform of the transmission function of the filter $(\alpha=40)$ is plotted in Fig. 10 as well. The radius of the illumination spot is assumed to be the same for both object positions. Therefore, the power spectra represent the two speckle patterns, characterized by speckle sizes of $\rho_{1}=8.9 \mu \mathrm{m}$ and $\rho_{2}=17 \mu \mathrm{m}$. According to Eq. (17), the total power of the photocurrent from the filter will be proportional to the product of the power spectrum of the filter function and the power spectrum of the speckle pattern [5]. Clearly, the product involving the power spectrum of the speckle pattern with a speckle size of $\rho_{1}$ collects contributions from significantly more rings than the product involving the power spectrum of the speckle pattern with the mean speckle size of $\rho_{2}$.

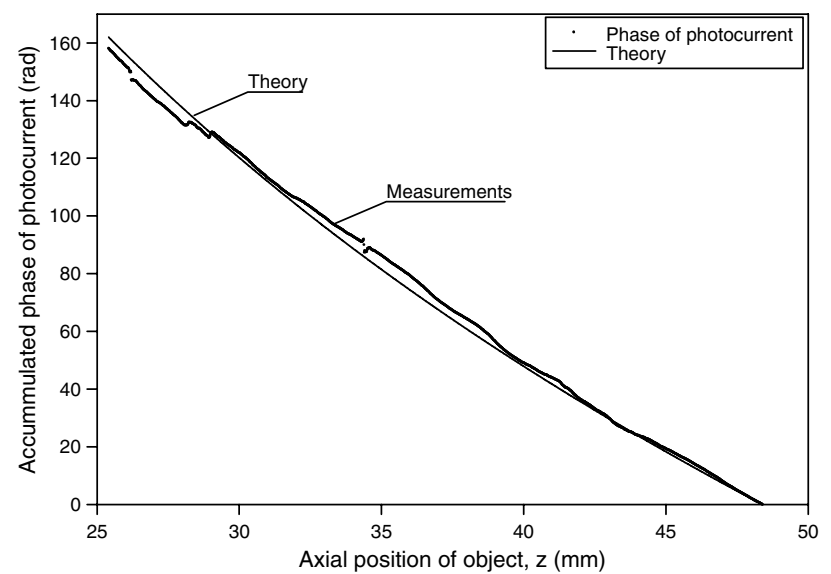

Fig. 9. Displacement is measured as the angle of the phasor based on the polar form of the two photocurrents in mutual phase quadrature. The angle is plotted as a function of the distance $z$ between the object and the filter. The filter [Eq. (22)] parameters are $\alpha=40$ for $r_{\mathrm{p}} \in[1 ; 512]$, while $t\left(r_{\mathrm{p}}\right)=0$ for $r_{\mathrm{p}}>474$. 


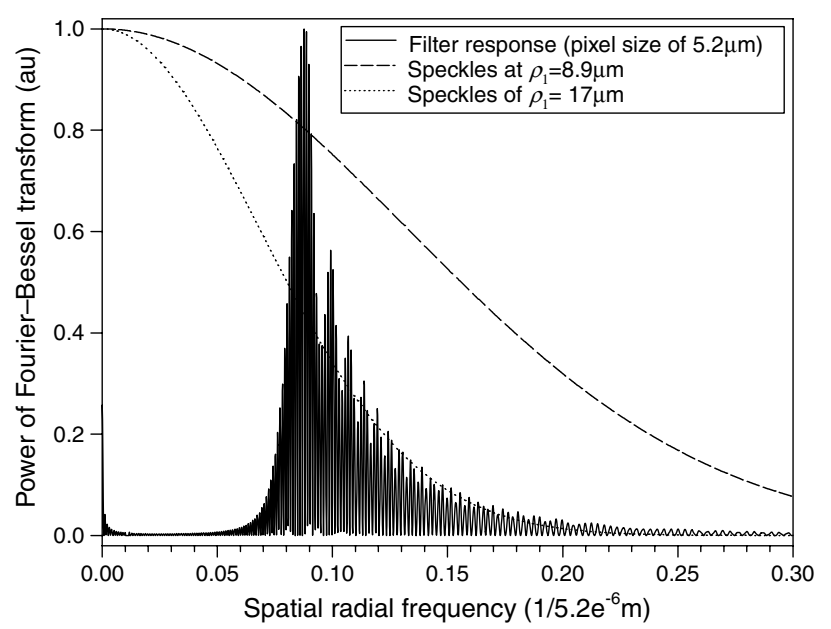

Fig. 10. Power of Fourier-Bessel transforms of the filter function [Eq. (21)] is illustrated together with the power spectra of the speckle patterns expected at $z=48.4 \mathrm{~mm}$ and $z=25.4 \mathrm{~mm}$. The filter parameters follows as $\alpha=40$ for $\alpha=40$, while $t\left(r_{\mathrm{p}}\right)=$ 0 for $r_{\mathrm{p}}>512$.

Therefore, the speckle size and its variation as a function of object position influence both the signal level and the selectivity of the filter.

A way to obtain a more uniform speckle size throughout the entire range of distances between the filter and the object is to illuminate the object with a laser beam, which diverges from a waist located at the center of the filter. The beam is emitted normal to the observation plane and beyond a distance compatible with the confocal parameter of the laser beam, the radius of the beam will increase linearly with the distance to the object. Therefore, according to Eq. (13) the speckle radius observed in the filter plane will remain constant and independent of the distance between the object and the filter. However, now the speckles will decorrelate as the radius of the beam varies as well.

The principle of the spatial filter technique is based on the light intensity. Therefore, the technique is applicable to incoherent illumination of objects as well. The principle of the filter relates to the scale and rotation invariance properties of the FourierMellin transform, used as an image processing technique for image recognition and distance measurements [18].

The benefits of the medium-precision technology appear when these filters can be implemented optically, and the photodetectors can be hardwired to produce the two photocurrents in real time [19]. In that case, the benefits are real-time processing of typically speckle correlation issues by optical spatial filtering velocimetry, and compact vibration or displacement sensor designs [20] can be produced at a low cost for industrial applications and navigational devices.

\section{Conclusion}

A spatial filter function has been designed for tracking objective speckle patterns expanding or contract- ing in the filter plane according to out-of-plane motion of an object, moving away from, or toward, the filter, respectively. The filter is designed to reduce the uneven contributions from the rings of various radii. The filters are emulated with a CCD camera, but applied to speckle images from real applications. Measurements of long range displacements $(23 \mathrm{~mm})$ are obtained giving a random error of $\pm 0.5 \mathrm{~mm}$. Measurements of short range out-of-plane vibrations are obtained with a noise-equivalent power corresponding to amplitudes of $1 \mu \mathrm{m}$.

\section{Appendix A}

The rotational symmetric expression in Eq. (21) is inserted into the Fourier-Bessel transform:

$$
G\left(f_{\mathrm{p}}\right)=\int_{0}^{R} r_{\mathrm{p}} \cos \left(2 \pi \alpha \ln \left(\frac{r_{\mathrm{p}}}{r_{0}}\right)+\theta_{0}\right) J_{0}\left(2 \pi r_{\mathrm{p}} f_{\mathrm{p}}\right) \mathrm{d} r_{\mathrm{p}}
$$

where $f_{\mathrm{p}}$ is the spatial radial frequency, the size of the filter is limited to a radius of $R$, and for simplicity $\theta_{0} \rightarrow 0$. After integration, the power of Eq. (A1) is found, and after additional reduction we find the power of the Fourier-Bessel transform of Eq. (21):

$$
\begin{aligned}
G\left(f_{\mathrm{p}}\right)= & \frac{R^{4}}{16\left(1+\pi^{2} \alpha^{2}\right)^{2}} \\
& \times \operatorname{Im}\left[\begin{array}{l}
(2 \pi \alpha-2 i) \exp \left(-i 2 \pi \alpha \ln \left(\frac{R}{r_{0}}\right)\right) \\
\times_{1} F_{2}\left(1-i \pi \alpha,\{1,2-i \pi \alpha\},-\pi^{2} R^{2} f^{2}\right)
\end{array}\right]^{2},
\end{aligned}
$$

where the hypergeometric function ${ }_{1} F_{2}(\cdot)$ is derived from the generalized hypergeometrical function ${ }_{\mathrm{p}} F_{q}(\cdot)[\underline{21]}:$

$$
{ }_{\mathrm{p}} F_{q}(\mathbf{a}, \mathbf{b}, z)=\sum_{k=0}^{\infty} \frac{\left(a_{1}\right)_{k} \ldots\left(a_{p}\right)_{k}}{\left(b_{1} \ldots\left(b_{q}\right)_{k}\right.} \frac{z^{k}}{k !} .
$$

The shape of the ensemble average speckle appears from spatial auto-covariance of the intensity distribution of the speckle patterns. The spatio-temporal covariance function equals the spatial autocovariance in the limit of $\tau \rightarrow 0$. Further, introducing the polar coordinate system to the auto-covariance function we find:

$$
R_{I}\left(r_{\mathrm{p}}\right)=\exp \left(-\frac{r_{\mathrm{p}}^{2}}{\rho_{0}^{2}}\right)
$$

Then, the power of the Fourier-Bessel transform can be found as:

$$
G\left(f_{\mathrm{p}}\right)=\int_{0}^{R} r_{\mathrm{p}} R_{I}\left(r_{\mathrm{p}}\right) J_{0}\left(2 \pi r_{\mathrm{p}} f_{\mathrm{p}}\right) \mathrm{d} r_{\mathrm{p}}=\frac{\rho_{0}^{2}}{2} \exp \left(-\pi^{2} \rho_{0}^{2} f_{\mathrm{p}}^{2}\right) .
$$




\section{References}

1. I. Yamaguchi, "Fringe formation in deformation and vibration and measurements using laser light," Prog. Opt. 22, 271-340 (1985).

2. M. Sjödahl, "Some recent advances in electronic speckle photography," Opt. Lasers Eng. 29, 125-144 (1998).

3. M. Sjödahl and H. O. Saldner, "Three-dimensional deformation field measurements with simultaneous TVholography and electronic speckle photography," Appl. Opt. 36, 3645-3648 (1997).

4. Y. Aizu and T. Asakura, Spatial Filtering Velocimetry, Fundamentals and Applications, Vol. 116 of Springer Series in Optical Sciences (Springer, 2005).

5. U. Schnell, J. Piot, and R. Dändliker, "Detection of movement with laser speckle patterns: statistical properties," J. Opt. Soc. Am. A 15, 207-216 (1998).

6. M. L. Jakobsen and S. G. Hanson, "Miniaturized lenticular array for laser speckle from solid surfaces," Meas. Sci. Technol. 15, 1949-1957 (2004).

7. N. Takai, T. Iwai, and T. Asakura, "Real time velocity measurements for a diffuse object using zero-crossing of laser speckle," J. Opt. Soc. Am. 70, 450-455 (1980).

8. T. F. Q. Iversen, M. L. Jakobsen, and S. G. Hanson, "Specklebased three-dimensional velocity measurement using spatial filtering velocimetry," Appl. Opt. 50, 1523-1533 (2011).

9. I. Yamaguchi and S. Komatsu, "Theory and applications of dynamic laser speckles due to in-plane object motion," Optica Acta 24, 705-724 (1977).

10. M. Giglio, S. Musazzi, and U. Perini, "Distance measurement from a moving object based on speckle velocity detection," Appl. Opt. 20, 721-722 (1981).
11. D. V. Semenov, E. Nippolainen, and A. A. Kamshillin, "Fast distance measurements by use of dynamic speckles," Opt. Lett. 30, 248-250 (2005).

12. J. W. Goodman, "Statistical properties of laser speckle patterns," in Laser Speckle and Related Phenomenon, J. C. Dainty, ed. (Springer-Verlag, 1984), pp. 9-75.

13. A. E. Siegman, Lasers (University Science, 1986).

14. H. T. Yura, B. Rose, and S. G. Hanson, "Dynamics laser speckle in complex ABCD optical systems," J. Opt. Soc. Am. A 15 1160-1166 (1998).

15. T. Yoshimura and S. Iwamoto, "Dynamic properties of three-dimensional speckles," J. Opt. Soc. Am. A 10, 324-328 (1993).

16. H. T. Yura, S. G. Hanson, R. S. Hansen, and B. Rose, "Threedimensional speckle dynamics in paraxial optical systems," J. Opt. Soc. Am. A 16, 1402-1412 (1999).

17. S. O. Rice, "Mathematical analysis of random noise," Bell Syst. Tech. J. 24, 46-159 (1945).

18. H. Zhang, L. Wang, R. M. Jia, and J. W. Li, "A distance measuring method using visual image processing," in Proceedings of the 2009 2nd International Congress on Image and Signal Processing (IEEE, 2009), Vols. 1-9, pp. 2275-2279.

19. N. Takai, T. Iwai, and T. Asakura, "Real time velocity measurements for a diffuse object using zero-crossing of laser speckle," J. Opt. Soc. Am. 70, 450-455 (1980).

20. S. G. Hanson, M. L. Jakobsen, H. C. Petersen, C. DamHansen, and J. Stubager, "Miniaturized optical specklebased sensor for cursor control," Proc. SPIE 6341, 63411U (2006).

21. S. Wolfram, "The Mathematica Book," 4th ed. (Wolfram Media/ Cambridge University, 1999). 